\title{
Node Localization Method for Wireless Sensor Networks Based on Hybrid Optimization of Differential Evolution and Particle Swarm Algorithm
}

\author{
Lieping Zhang ${ }^{1,2, *}$, Wenjun $\mathrm{Ji}^{2}$ and Yu Zhang ${ }^{1,2}$ \\ ${ }^{I}$ GuangXi Key Laboratory of New Energy and Building Energy Saving, Guilin University of Technology, Guilin 541004, \\ China \\ ${ }^{2}$ College of Mechanical and Control Engineering, Guilin University of Technology, Guilin 541004, China
}

\begin{abstract}
Regarding the node localization problems for wireless sensor network, a hybrid optimization method was proposed accordingly on differential evolution(DE) algorithm and particle swarm optimization(PSO) algorithm. Firstly, the position and velocity of the initial population were randomly generated by $\mathrm{PSO}$, and the fitness function was constructed according to the mean square error of estimated and measured distance between the unknown nodes and their adjacent anchor node. Secondly, the mutation and selection operation of DE algorithm were executed to find out the optimum position of the population. Lastly, the current velocities and positions of all particles of the population were updated, and the crossover operation and selection operation of DE algorithm were executed to update the current global optimum position of the whole population. Population global optimum solution of iterative search algorithm is the position coordinate of the unknown node. Simulation results indicate that the proposed localization method has smaller average localization error and higher localization accuracy than that of DE algorithm and PSO algorithm in the same environment.
\end{abstract}

Keywords: Wireless sensor network, Differential evolution algorithm, Particle swarm optimization algorithm, Hybrid optimization, Node localization.

\section{INTRODUCTION}

Position information is of great importance to wireless sensor network (WSN) monitoring activities. Event occurring place or node position of acquired information accounts for the important information that contains in wireless sensor node monitoring information [1]. In nature WSN localization is equivalent to optimizing different measured distances or routes, so it is a NP unsolvable problem [2]. In the meanwhile, as node localization method based on optimization algorithm has the features of non-dependence on the adopted localization method, high localization accuracy, etc., researchers proposed the method to improve the performance of WSN node localization by using the optimization algorithm. Ewa Niewiadomska-Szynkiewicz and others made a study over WSN node localization method by adopting heuristic algorithm, such as simulated annealing and genetic algorithm, which in turn proves the node localization effectiveness of heuristic algorithm [3]. Chehri A. and others proposed a non-linear optimization localization algorithm based on differential evolution(DE) algorithm [4], which has the advantage of low complexity. But the method has the advantages of low localization accuracy if the measured distance error is big, node wireless range is small or anchor node ratio is low. Wuling Ren and others proposed a localization algorithm based on shuffled frog leaping algorithm and particle swarm optimization(PSO)algorithm [5], which has a good localization accuracy. The method has a good localization effect, notably if the measured distance error is big enough, but its consumption and stability remain to be improved. A new localization algorithm for WSN by combining extremum disturbed and simple particle swarm optimization was proposed to solve the distance estimation errors problem of DV-hop algorithm [6]. A group particle swarm optimization algorithm was proposed and used to optimize the result of the maximum likelihood estimation method based on time of arrival(TOA) [7].

$\mathrm{DE}$ algorithm is one algorithm based on population evolution proposed by R. Storn and K. Price [8]. It offers solution to the optimum question through cooperation and competition among population individuals and it has the advantages of simplification, high-efficiency, high convergence rate, good robustness, and so on. PSO algorithm is a kind of self-adaptive global optimization heuristic algorithm that has the advantages of simple algorithm, easy adoption, fast search,and son on [9]. However, as a single algorithm,DE algorithm is also limited to such problems as local convergence, slow late convergence rate and unstable performance when solves complicated optimization question, and PSO algorithm is limited to confined search range, vulnerability to local optimization and other problems [10]. Obviously PSO algorithm with good optimization and DE algorithm with strong strength in maintaining population diversification and searching ability are well complementary to each other [11]. Therefor, a hybrid-optimized WSN node localization method based on DE algorithm and PSO algo- 
rithm is proposed in this paper,and its effectiveness is proved through simulation experiments.

\section{DESCRIPTION OF LOCALIZATION PROBLEMS}

WSN node localization problem is actually the process that position coordinates of unknown nodes are achieved by the distance information, wireless communications radius according to some localization strategy [12]. Since there is an error in distance measurement technology, measured distance between anchor node and unknown node is not the real value. Therefor, position estimation of unknown node can be treated as a kind of optimization which minimizes the target function of localization error of unknown node to find out the position coordinate of unknown node. The ranging error is an important factor affecting the unknown node location error, and reduce the maximum error can effectively improve the localization accuracy. The mean square error of distance between unknown node and adjacent anchor node is defined as the fitness function of localization problem. As shown below:

$f_{i}(\hat{x}, \hat{y})=d_{i}-\sqrt{\left(x_{i}-\hat{x}\right)^{2}+\left(y_{i}-\hat{y}\right)^{2}}$

Wherein, $(\hat{x}, \hat{y})$ is the estimated coordinate of unknown node, $\left(x_{i}, y_{i}\right)(\mathrm{i}=1,2, \ldots, \mathrm{M})$ is the actual coordinate of the ith anchor node, $\sqrt{\left(x_{i}-\hat{x}\right)^{2}+\left(y_{i}-\hat{y}\right)^{2}}$ is the estimated distance between the ith anchor node and that unknown node,

$f_{i}(\hat{x}, \hat{y})$ is the error value between measured and estimated distance of the ith anchor node and that unknown node, and $d_{i}$ is the measured distance between the ith anchor node and that unknown node. As a matter of fact, measured distance between two nodes is not the actual distance in reality, so the combined form of actual distance and gaussian error should be used for measuring distance [4], namely:

$d_{i}=d_{i j}(1+\operatorname{rand} n \times \eta)$

Wherein, $\mathrm{d}_{\mathrm{ij}}$ is the actual value between two nodes, $d_{i j}=\sqrt{\left(x_{i}-x_{j}\right)^{2}+\left(y_{i}-y_{j}\right)^{2}}, \eta$ is the error factor related to distance measurement accuracy, randn is the random variable subject to standard normal distribution, where the average value is ' 0 ' and square variance is ' 1 '.

In this way, WSN node localization problem is converted to the fitness function optimization problem and the optimum solution provided by minimizing fitness function is therefore the estimated position of unknown node. The improving localization error fitness function can be shown as below, that the weighted position information of nearest hop distance between anchor node and the unknown node are introduced to the primary fitness function. As shown below:

$$
F_{i}(\hat{x}, \hat{y})=\sum_{i=1}^{M} \alpha_{i}^{2} f_{i}^{2}(\hat{x}, \hat{y})
$$

Wherein, $\mathrm{M}$ is the number of nearest hop distance of anchor node to the unknown node, and in this paper,
$\mathrm{M}=4$, which is the minimum number of $\mathrm{M} . \alpha_{\mathrm{i}}$ is the accuracy weight of measured distance between unknown node and the ith anchor node, which is in reverse proportion with the shortest route hop count between unknown node and the ith anchor node. The hop count can be calculated by Dijkstra algorithm. Improved fitness function can effectively reduce algorithm complexity, running time and node energy consumption but extend network lifetime. Optimization algorithm can be adopted to optimize the fitness function, find out the optimum solution and improve node position accuracy.

\section{DESCRIPTION OF NODE LOCALIZATION METHOD}

\subsection{DE Algorithm}

Similar to genetic algorithm, DE algorithm repeats iterative computation from one randomly generated initial swarm in accordance with some operation rules, such as selection, hybridization and mutation, and guides the search close to the optimum solution by surviving good individuals while excluding the bad ones in line with every individual fitness value. However, different from genetic algorithm, DE algorithm uses differential strategy in operating mutation intended for individual mutation concretely through disturbing individuals by use of differential vectors among population individuals. DE algorithm can be extended as follows [13].

Step 1: Initialized population. Define population scale as $\mathrm{NP}$ and maximum iteration as $\mathrm{t}_{\mathrm{max}}$, and randomly generate the initial population meeting restraint condition in $\mathrm{D}$ dimensional space.

In which, $\mathrm{i}=1,2, \ldots, \mathrm{NP}, \mathrm{j}=1,2, \ldots, \mathrm{D}$, $_{\text {and }}(0,1)$ stands for random number evenly distributed in the interval $(0,1) \cdot b_{j, U}$, $b_{j, L}$ respectively stands for the $X_{j, i}$ upper and bottom limit.
As shown below:

$X_{j, i, 0}=\operatorname{rand}_{j}(0,1) \times\left(b_{j, U}-b_{j, L}\right)+b_{j, L}$

Step 2: Mutation operation. Mutation is the key step of $\mathrm{DE}$ algorithm and the common strategy of $\mathrm{DE}$ algorithm is intended for changing through mutation operation. The individual generation strategy of $\mathrm{DE}$ algorithm can be expressed as shown: $\mathrm{DE} / \mathrm{x} / \mathrm{y} / \mathrm{z}$. Wherein, ' $\mathrm{x}$ ' stands for mutated individual vector type, ' $y$ ' stands for differential vector quantity and ' $\mathrm{z}$ ' stands for crossover method. In this paper, ' $\mathrm{DE} / \mathrm{rand} / \mathrm{l} / \mathrm{bin}$ ' strategy is used in the mutation operation, which is a widely used combination strategy. Mutation operation for Gth generation evolved target vector $X_{i, G}(i \in[1$, $\mathrm{NP}]$ ) is shown as below:

$V_{i, G+1}=X_{r 1, G}+F \times\left(X_{r 2, G}-X_{r 3, G}\right)$

Step 3: Crossover operation. Mutation vector will randomly change with the target vector. when a new vector 'experiment vector' $U_{j, i, G+1}$ would be output after crossover. Target vector $X_{i}$ and mutation vector $V_{i}$ in iteration $\mathrm{j}$ are defined in the following equation. As shown below: 


$$
U_{j, i, G+1}=\left\{\begin{array}{c}
V_{j, i, G+1} \text { if }(\text { rand } \leq C R) \operatorname{or}(j=\operatorname{rnbr}(i)) \\
X_{j, i, G+1} \text { if }(\text { rand }>C R) \text { and }(j \neq \operatorname{rnbr}(i))
\end{array}\right.
$$

Step 4: Selection operation. Through the above operations combined comparing fitness values of the experiment vector and target vector, the population is therefore selected to be passed on to the next generation. As shown below:

$$
X_{i, G+1}=\left\{\begin{array}{c}
U_{i, G+1} \text { if } F\left(U_{i, G+1}\right) \leq F\left(X_{i, G}\right) \\
X_{i, G} \text { otherwise }
\end{array}\right.
$$

\subsection{Particle Swarm Optimization Algorithm}

PSO algorithm takes every individual as particle without any volume and weight in the search space. Every particle within the search space has its own position and velocity as well as fitness value determined by optimization function through regulating operation rules for each particle. Particle flies at one velocity in the search space, during which the optimum position the particle once passed is the optimum solution searched by this particle. All particles are searching within the solutions space of the optimum particle, and gradually the optimum solution is searched out through iterative search, or called as individual best value, namely $\mathrm{P}_{\text {best }}$. The best position that the entire population (or some particles) once passed is the optimum solution found out to the entire population (or some particles), or called as global (or local) best value, namely $\mathrm{G}_{\text {best }}$. Particle will be updated with individual best value $P_{\text {best }}$ and global (or local) best value $G_{\text {best }}$ during every iteration. In the population composed of $m$ particles within the $n$-dimensional space, the ith particle $X_{i}$ will change its own velocity and position based on equation (8) and (9).

$$
\begin{aligned}
& V_{i d}(t+1)=w \times V_{i d}(t)+C_{1} \times \operatorname{rand}() \times\left(P_{i d}-X_{i d}\right)+ \\
& C_{2} \times \operatorname{rand}() \times\left(P_{g d}-X_{i d}\right) \\
& X_{i d}(t+1)=X_{i d}(t)+V_{i d}(t+1)
\end{aligned}
$$

Among which, $1 \leq i \leq m, 1 \leq d \leq n$, and $\mathrm{t}$ is the number of iteration for current population, and $\mathrm{w}$ is the inertia weight, $\mathrm{C}_{1}$ and $\mathrm{C}_{2}$ are positive constants called as learning factors, and $\operatorname{rand}()$ is the random number evenly distributed in the interval $[0,1]$.

\subsection{WSN Node Localization Method Based on Hybrid Optimization of Differential Evolution and Particle Swarm Algorithm}

DE algorithm and PSO algorithm are both random heuristic algorithms based on swarm intelligence. The evolution process with randomness would result in some blindness in search for the optimum solution. Notably in offering solution to complex optimization question, DE Algorithm is limited to the convergence rate of solving complicated optimization in the late optimization iteration, which would easily result in local optimization. As for PSO algorithm, premature convergence appearing as a result of reduced particle diversification would also easily result in local optimization. In order to cover defects for both DE algorithm and PSO algorithm, a hybrid optimization algorithm of differential evolution and particle swarm algorithm (DEPSO) would be proposed through an effective combination of $\mathrm{DE}$ algorithm and PSO Algorithm. This algorithm adds disturbance on the current position of PSO particle by use of DE algorithm mutation operation for the benefit of maintaining swarm diversification, improving PSO algorithm space search capacity and avoiding particle into local optimization.

As hybrid optimization DEPSO algorithm features better global convergence, it would improve node localization accuracy by applying it to WSN node localization. In this way, this paper intends to propose a WSN node localization method based on DEPSO algorithm. Firstly, it randomly generates the initial swarm, initializes all individual positions and velocities, calculates and stores individual best value $P_{\text {best }}$ as well as global optimum solution $\mathrm{G}_{\text {best }}$, executes mutation and selection operation for differential evolution, and eventually finds out the swarm optimum position. Secondly, it renews velocities and positions of all particles in the swarm by using PSO velocity and position equation. Thirdly, it renews the swarm optimum position by use of crossover and selection operation of DE algorithm. Lastly, it outputs the global optimum solution for swarm until all iteration termination conditions are met or maximum iterations are reached. The position of particle in the search space through PSO algorithm is the position of unknown node, swarm global optimum solution renewed through algorithm iteration is the particle optimum position, or estimated position coordinates for the unknown node, and the estimation process of unknown node position is the process of minimizing localization error fitness function. The node localization design mentioned in this paper is based on DEPSO hybrid algorithm, which is specified as follows:

Step 1: Initial parameter setup for algorithm: swarm scale $N P$, maximum iterations $t_{\max }$, scaling factor $F$, crossover rate $\mathrm{CR}$, maximum velocity $\mathrm{v}_{\max }$, learning factor $\mathrm{C}_{1}$ and $\mathrm{C}_{2}$, initial inertia weight $\mathrm{w}_{\max }$, final inertia weight $\mathrm{w}_{\min }$, iterations $\mathrm{t}=0$, and error factor $\eta$.

Step 2: Initialized swarm: randomly deploy $\mathrm{N}$ unknown nodes and $\mathrm{M}$ anchor nodes within the specific network area, randomly generate the initial position and velocity for each particle, calculate the fitness value for each particle and set the current particle position as individual best value $\mathrm{P}_{\text {best }}$, among which the best is kept as global best value $\mathrm{G}_{\text {best }}$.

Step 3: Calculate the distance matrix D as composed of the respective distance between $\mathrm{N}$ unknown nodes to $\mathrm{M}$ anchor nodes.

Step 4: Calculate the shortest route and hops between each unknown node and anchor node by Dijkstra algorithm, and find out the distance matrix $d$ and its hops $\mathrm{N}_{\text {hop }}$ between the unknown node $\mathrm{i}$ and its 4 nearest anchor nodes. It can be converted to find out the node position $[\hat{x}, \hat{y}]$ that minimizes the fitness function by use of DEPSO algorithm, equivalent to solve $[\hat{x}, \hat{y}]=\arg \min \left(F_{i}(\hat{x}, \hat{y})\right)$. 


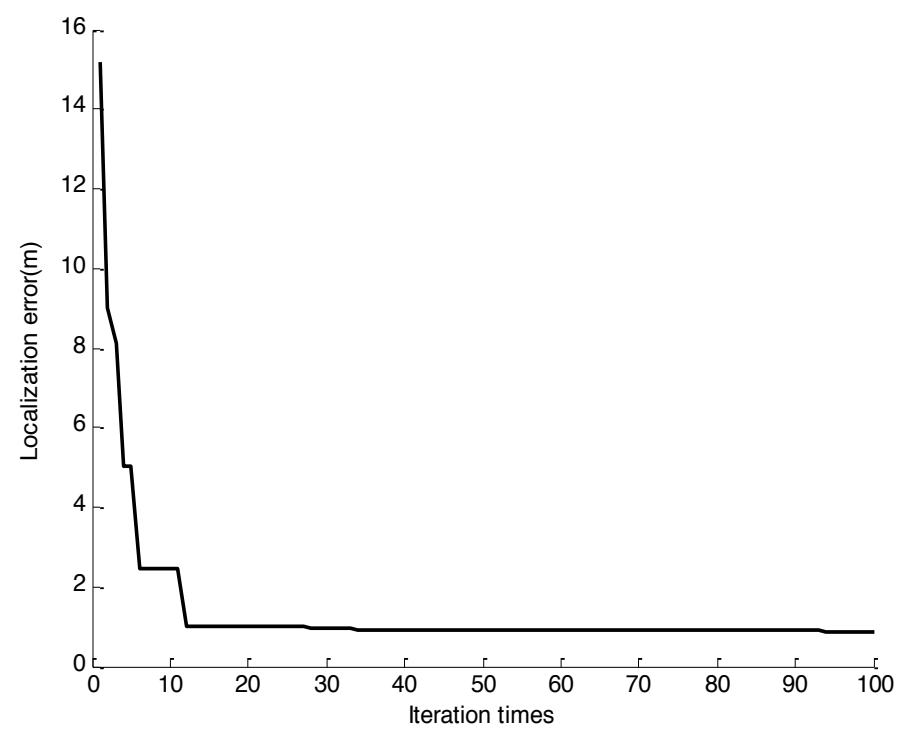

Fig. (1). When error factor $\eta=5 \%$, the influence of iteration times on localization error.

Step 5: Parameter input: anchor node coordinate matrix Beacon, distance matrix $d$, and weight $\alpha_{I}$.

Step 6: Iterations $\mathrm{t}=\mathrm{t}+1$.

Step 7: Proceed on mutation and selection operation for the swarm in line with equation (6) and equation (8).

Step 8: Calculate fitness function value for each individual by use of equation (4), and compare it with the optimum solution to current historic swarm, among which the fitness function with the smallest value shall be passed to the next generation, equivalent to renew the optimum position for current swarm.

Step 9: Renew the positions and velocities of all individuals in the swarm in line with equation (9) and equation (10).

Step 10: Execute crossover and selection operation for each individual position in line with equation (7) and equation (8), recalculate the fitness values for all individuals and compare corresponding fitness value for current optimum position and the fitness value for swarm historic optimum position, among which the individual with the smallest fitness value shall be passed to the next generation, namely this individual position is the optimum position of the current swarm.

Step 11: Judge if the maximum iteration $t_{\max }$ is reached or not, if so, it outputs the corresponding individual position $[\hat{x}, \hat{y}]$ with global optimum solution, or the estimated coordinate for unknown node position, or otherwise return to Step 6.

\section{SIMULATION EXPERIMENT AND ITS RESULT ANALYSIS}

\subsection{Setup of Simulation Environment}

Simulation experiment is carried out in MATLAB. Set the total network nodes as 100 randomly distributed in $100 \mathrm{~m}$ $\times 100 \mathrm{~m}$ square area, where unknown nodes and anchor nodes are randomly generated and deployed. Through experience and many repeated experiments to determine the simulation parameter by DEPSO localization method: node unlimited range $\mathrm{R}=40 \mathrm{~m}$, swarm scale $\mathrm{NP}=20$, maximum iterations $\operatorname{tmax}=100$, maximum velocity $\mathrm{v}_{\max }=6$, initial inertia weight $\mathrm{w}_{\max }=0.9$, final inertia weight $\mathrm{w}_{\min }=0.4, \mathrm{C}_{1}=\mathrm{C}_{2}=2$, scaling factor $\mathrm{F}=0.5$, crossover rate $\mathrm{CR}=0.6$, and let error factor as $\eta=5 \%$. Compare average localization error from the perspective of algorithm iterations, anchor node density, distance measurement error and anchor wireless range by employing three methods DE, PSO and DEPSO through 100 simulation experiments.

\subsection{Simulation Result and tts Analysis}

DEPSO localization method has been proved to be a kind of localization method with high localization accuracy that only needs a few anchor nodes and smaller node wireless range by correspondingly changing iterations, anchor node density, network connectivity and distance measuring error. Compare DEPSO localization method with DE localization method and PSO localization method for analyzing DEPSO localization method performance. The average localization error equation used to evaluate DEPSO localization method performance in simulation is shown as below:

$$
\text { error }=\frac{100}{N \times R} \sum_{i=1}^{N} \sqrt{\left(x_{i}-\hat{x}_{i}\right)^{2}+\left(y_{i}-\hat{y_{i}}\right)^{2}} \%
$$

Among which, $\mathrm{R}$ is node communications radius, $\mathrm{N}$ is the number of unknown nodes, $\left(\hat{x}_{i}, \hat{y}_{i}\right)$ is the estimated position of the unknown node $\mathrm{i}$, and $\left(x_{i}, y_{i}\right)$ is the actual position of the unknown node $\mathrm{i}$.

Algorithm convergence is the key to algorithm effectiveness. Fig. (1) shows the correlation between iteration and localization error by DEPSO hybrid optimization method when the error factor $\eta=5 \%$. It follows that the localization 


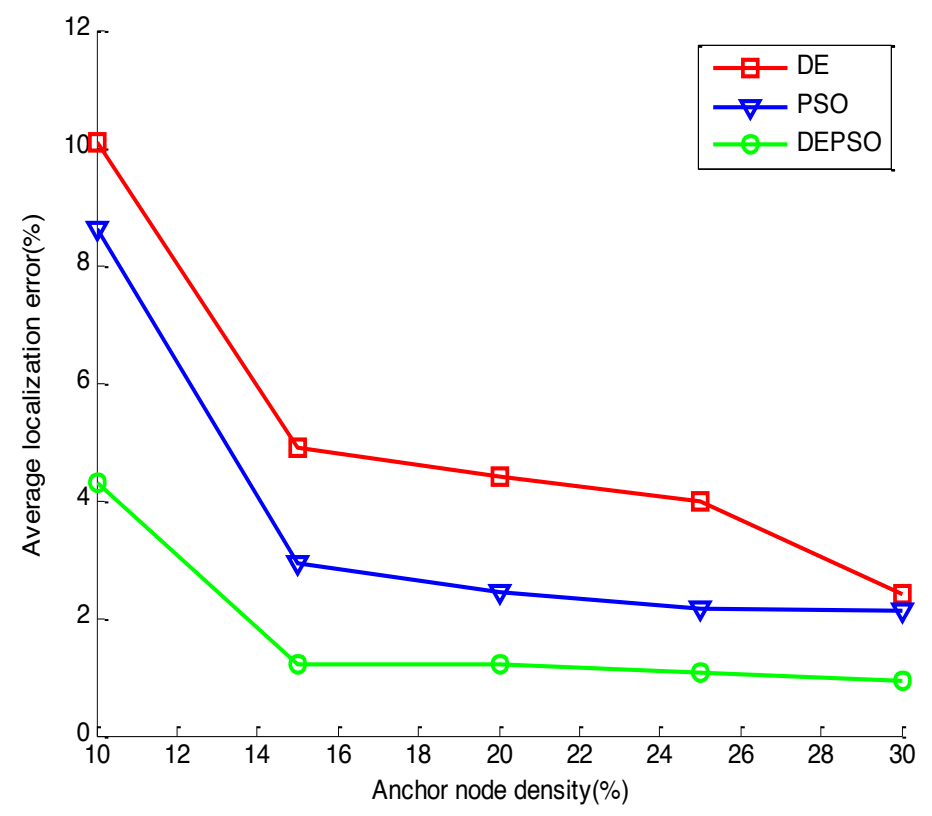

Fig. (2). The influence of anchor node density on average localization error.

error by DEPSO localization method also continuously decreases with the increase of iterations if there is distance measurement error. When the iterations reach 20, localization error curve tends to be flat with the localization error converging approximately at $1.0 \mathrm{~m}$. Consequently, DEPSO method featuring strong convergence can be used to accurately estimate the position of unknown node.

The proportion of anchor nodes is another important index for evaluating localization effects, because the number of anchor nodes directly determines the consumption. In simulation experiments, the anchor node density increase from $10 \%$ to $30 \%$ at $5 \%$ interval if other parameters stay unchanged; the influence of changing anchor node density upon the average localization error using all localization methods is observed. Fig. (2) shows the influence of anchor node density on average localization error. Clearly, the average localization errors by use of these three methods decrease with the increase of anchor node density, but DEPSO hybrid optimization method has higher localization accuracy at the same anchor node density or with the same anchor nodes. In other words, anchor nodes quantity used by DEPSO localization method is the lowest under the requirement of the same localization accuracy. However the quantity of anchor nodes directly influences the network cost and energy consumption, the overall performance of DEPSO localization method featuring the lowest cost and consumption is better than other two localization methods.

Network connectivity refers to the quantity of adjacent nodes of unknown nodes. Generally, strong network connectivity will bring about more localization information used by nodes, thus improving node localization accuracy and node localization rate. Simulation could change network connectivity by changing the wireless range of nodes. Therefore the wireless range of anchor nodes is used as one performance index to evaluate localization error. In simulation experiment, network connectivity is changed with the change of anchor node wireless range $\mathrm{R}$ if node-deploying area and other parameters stay unchanged. This paper observes the localization error by setting anchor node wireless range $\mathrm{R}$ increase from $20 \mathrm{~m}$ to $45 \mathrm{~m}$ at $5 \mathrm{~m}$ interval using all kinds of methods. Fig. (3) compares the influence of anchor node radio range $\mathrm{R}$ on the average localization error respectively based on DE, PSO and DEPSO localization method. From which the average localization errors based on these three methods invariably decrease with the increase of anchor node wireless range.In the same anchor node radio range, the localization error based on DEPSO algorithm is the lowest while node radio range corresponds to node transmitting power. Consequently, DEPSO localization method could be used to satisfy localization accuracy and extend network lifetime with a slower variation tendency of error curve, thus verifying its better stability and robustness than other two methods.

In view of the inaccuracy regarding disturbance and distance measuring technology in the external environment, there is unavoidable measured distance error between anchor node and unknown node. In simulation experiment, the proposed localization method and relevant performance are compared through changing error factor $\eta$ when other parameters stay unchanged. Fig. (4) shows the influence of distance error on average localization error when the error factor $\eta$ increases from $0 \%$ to $20 \%$ at $5 \%$ hop length. From it the average localization errors of three methods respectively increase with the increase of distance measurement error, but DEPSO algorithm has better performance than other two methods. With the same distance measurement error, the 


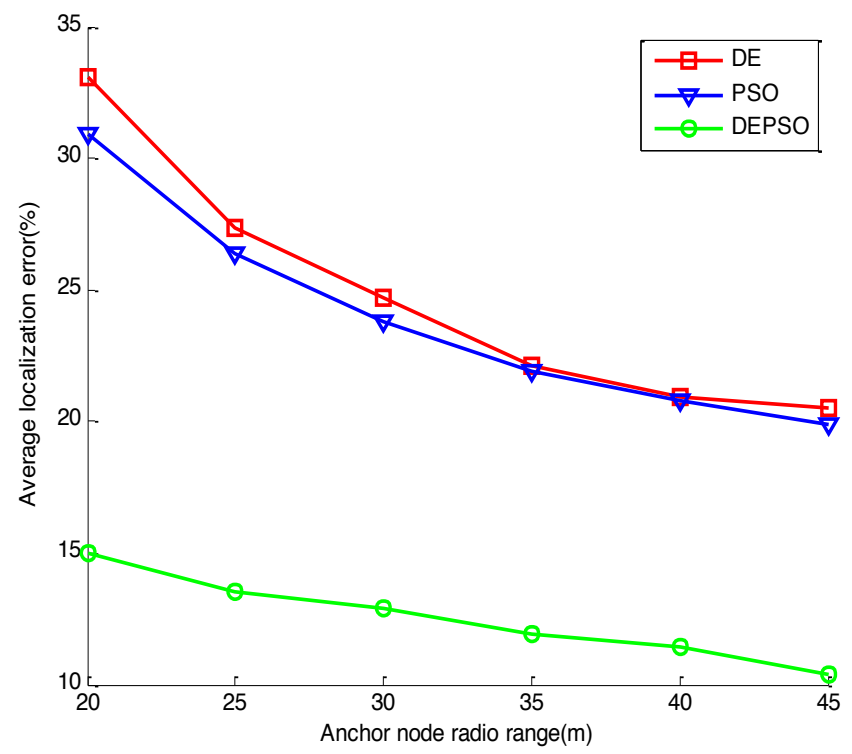

Fig. (3). The influence of anchor node radio range on average localization error.

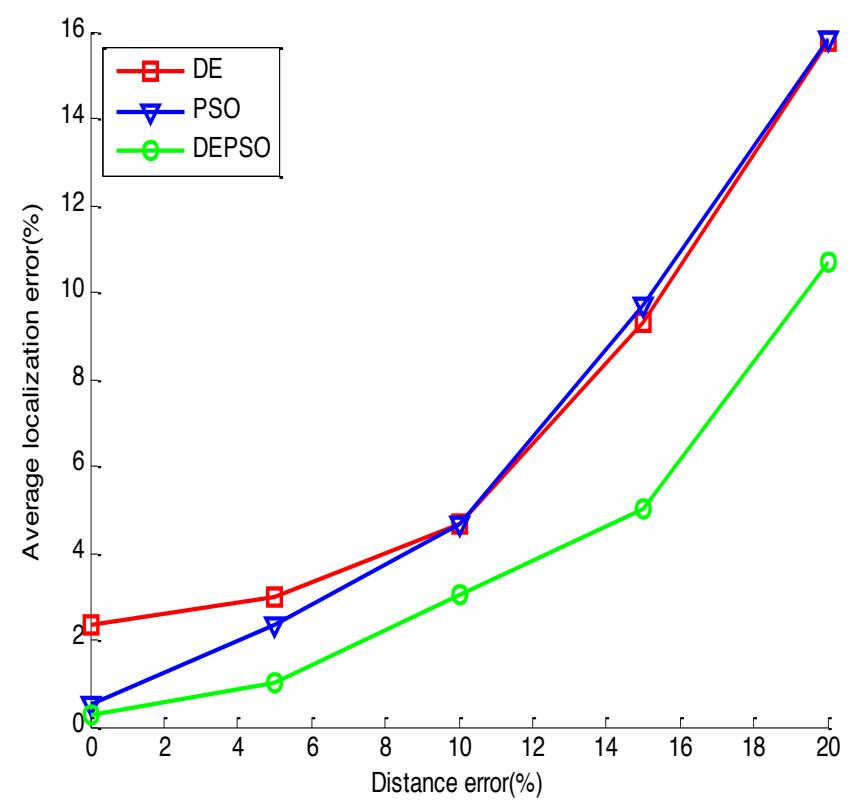

Fig. (4). The influence of distance error on average localization error.

localization error based on DEPSO algorithm is the lowest, and its average localization error curve slope is also the lowest among these three methods. In other words, this localization method with better localization effect receives the minimal influence of distance measurement error.

For the convenience of comparing three localization effects, the unknown node localization effects by three methods are put under the uniform ordinate scale for comparison as shown in Fig. (5), under the condition that the initial chosen anchor node density is $20 \%$, and network unknown nodes amounts to 80 . From it the error curve fluctuation for unknown nodes based on PSO algorithm is much weaker than that of DE algorithm, proving PSO algorithm has better stability than DE algorithm. However, both DE method and PSO method has the single case of bigger node localization error, proving local minimal value when localization at this node. Among three localization error variation curve, DEPSO localization method features lowest error, and its 


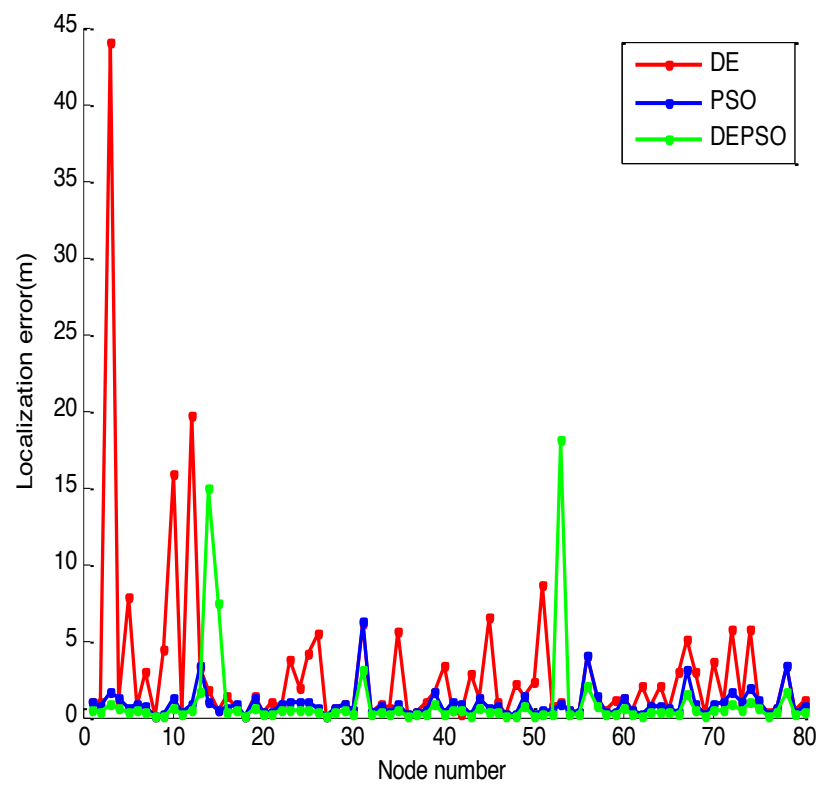

Fig. (5). The comparison of node localization errors of three localization methods.

fluctuation range and variation tendency of localization error curve are lower than that of other two methods. Although there is single case of bigger localization error, it nearly can be ignored when compared with the above two localization methods. Overall speaking, the localization method proposed in this paper featuring higher localization accuracy and better global convergence would effectively prevent the occurrence of local optimum solution.

\section{CONCLUSION}

This paper intends to propose a hybrid-optimized method for WSN node localization by adopting an effective strategy and analyzing the respective characteristics of DE algorithm and PSO algorithm. Reducing the square error of estimated and measured distance between the unknown node and its adjacent anchor node can guarantee a better localization accuracy. Simulation results prove that this method features smaller localization error, higher localization accuracy and better stability performance, but it also requires more calculation quantity while improving the accuracy. In this concern, future researches will focus on how to minimize the node energy consumption and extend network lifetime.

\section{CONFLICT OF INTEREST}

The author confirms that this article content has no conflict of interest.

\section{ACKNOWLEDGEMENTS}

This work was supported by the key project of Guangxi educational science foundation (No.201202ZD051), the research fund of Guangxi key laboratory of new energy and building energy saving (No.12-03-21-3), and the doctoral research initial foundation of Guilin University of Technology.

\section{REFERENCES}

[1] P. K. Singh, B. Tripathi, and N. P. Singh, "Node Localization in Wireless Sensor Networks", International Journal of Computer Science and Information Technologies, vol. 2, no. 6, pp. 25682572, 2011.

[2] J. Aspnes, D. Goldenberg, and Y. R. Yang, "On the Computational Complexity of Sensor Network Localization", In: Proceedings of First International Workshop on Algorithmic Aspects of Wireless Sensor Networks, pp. 32-44, 2004.

[3] E. N. Szynkiewicz, M. Marks, and M. Kamola, "Localization in wireless sensor networks using heuristic optimization techniques", Jouranl of Telecommunication and Information Technology, vol. 4, pp. 55-64, 2011.

[4] A. Chehri, P. Fortier, and P. M. Tardif, "Geo-location with wireless sensor networks using non-linear optimization", International Journal of Computer Science and Network Security, vol. 8, no. 1, pp. 145-154, 2008.

[5] W. Ren, and C. Zhao, "A localization algorithm based on SFLA and PSO for wireless sensor network", Information Technology Journal, vol. 12, no. 3, pp. 502-505, 2013.

[6] Q. Zhang, and M. Cheng, “ A node localization algorithm for wireless sensor network based on improved particle swarm optimization", Lecture Notes in Electrical Engineering, vol. 237, pp. 135144, 2014.

[7] D. Feng, and X. Jiang, "WSN node localization technology research based on GPSO", Computer Simulation, vol. 31, no. 2, pp. 370-373, 2014.

[8] R. Storn, and K. Price, "Differential evolution-a simple and efficient heuristic for global optimization over continuous spaces", Journal of Global Optimization, vol. 11, pp. 341-359, 1997.

[9] J. Kennedy, and R. C. Eberhart, "Particle Swarm Optimization", In: Proceedings of IEEE International Conference on Neural Networks, 1942-1948, 1995

[10] Y. Chi, J. Fang, and G. Cai, "Hybrid optimization algorithm based on differential evolution and particle swarm optimization", Computer Engineering and Design, vol. 30, no. 12, pp. 2963-2965, 2009. 
[11] B. Xin, and J. Chen, "A survey and taxonomy on hybrid algorithms based on particle swarm optimization and differential evolution", Journal of System Science and Mathematical Science, vol. 31, no. 9, pp. 1130-1150, 2011.

[12] S. Zhao, M. Sun, and Y. Tang, "GASA-Based localization algorithm for wireless sensor networks", Computer Applications and Software, vol. 26, no. 10, pp. 189-192, 2009.
[13] A. K. Qin, V. L. Huang, and P. N. Suganthan, "Differential evolution algorithm with strategy adaptation for global numerical optimization", IEEE Transactions on Evolutionary Computation, vol. 13, no. 2, pp. 398-414, 2009.

(C) Zhang et al.; Licensee Bentham Open.

This is an open access article licensed under the terms of the Creative Commons Attribution Non-Commercial License (http://creativecommons.org/licenses/by-nc/3.0/) which permits unrestricted, non-commercial use, distribution and reproduction in any medium, provided the work is properly cited. 